\title{
Padrão epidemiológico da má oclusão em pré-escolares brasileiros
}

\author{
Epidemiological pattern of malocclusion in Brazilian preschoolers
}

José Mansano Bauman ${ }^{1}$

João Gabriel Silva Souza ${ }^{2}$

Claudiana Donato Bauman ${ }^{3}$

Flávia Martão Flório ${ }^{1}$

${ }^{1}$ Departamento de Saúde Coletiva, Faculdade São Leopoldo Mandic. R. José Rocha Junqueira 13, Ponte Preta. 13041-445 Campinas SP Brasil.

jmbauman@gmail.com

${ }^{2}$ Departamento de Ciências Fisiológicas, Faculdade de

Odontologia de Piracicaba Universidade Estadual de

Campinas. Piracicaba SP

Brasil.

${ }^{3}$ Programa de Pós-

Graduação em Ciências da Saúde, Universidade Estadual de Montes Claros. Montes Claros MG Brasil.
Abstract This study aimed to evaluate the prevalence and distribution profile of malocclusion in Brazilian preschoolers and its association with macro-region, housing, gender, and self-reported race. In total, 6,855 children aged five years participating in the National Oral Health Survey (called SB Brasil 2010) were analyzed. Malocclusion was diagnosed according to the Foster and Hamilton index. We conducted descriptive, bivariate and multiple regression analyses (PR/CI95\%). We identified that $63.2 \%$ of children had at least one of the occlusal problems evaluated: canines' key $(22.9 \%)$, overjet (32.9\%), overbite (34.6\%), and posterior crossbite (18.7\%) and, thus, were considered with malocclusion. Higher probability of the presence of malocclusion was identified among the residents of the Midwest $(1.08 / 95 \% C I$ 1.01-1.15), Northeast (1.21/95\%CI 1.14-1.28), Southeast (1.27/95\%CI 1.20-1.34) and South (1.34/95\%CI 1.26-1.42) regions when compared to residents in the North. It was also higher among female children (1.06/95\% CI 1.02-1.09). No associations were identified concerning race and location of the municipality (capital/no capital). A high prevalence of malocclusion was identified in Brazilian preschoolers, and it was associated with gender and the macro-region. These findings may contribute to expanded public policies and greater access to treatment for this population.

Key words Malocclusion, Child, Preschool, Oral health, Prevalence
Resumo Objetivou-se avaliar a prevalência e o padrão de distribuição da má oclusão em pré-escolares brasileiros e sua associação com macrorregião, local de moradia, sexo e raça autodeclarada. Foram analisados dados de 6.855 crianças de 5 anos participantes do SB Brasil 2010. No levantamento, a má oclusão foi diagnosticada segundo o Indice de Foster e Hamilton. Conduziu-se análises descritivas, bivariadas e múltiplas (RP/ IC95\%). Identificou-se que 63,2\% das crianças possuíam ao menos um dos problemas oclusais avaliados: chave de caninos (22,9\%), sobressaliência (32,9\%), sobremordida (34,6\%), e mordida cruzada posterior $(18,7 \%)$ e, portanto, foram considerados com má oclusão. Maior chance da presença de má oclusão foi identificada entre os residentes das regiões Centro-Oeste (1,08/IC95\%1,01-1,15), Nordeste (1,21/IC95\%-1,14-1,28), Sudeste (1,27/IC95\%-1,20-1,34) e Sul (1,34/ IC95\%-1,26-1,42), quando comparados aos residentes na região Norte. Foi maior também entre as crianças do sexo feminino (1,06/IC95\%-1,021,09). Identificou-se elevada prevalência de má oclusão em pré-escolares brasileiros, sendo esta associada ao sexo e à macrorregião de moradia. Tais achados podem contribuir na ampliação de políticas públicas e no acesso ao tratamento para esta população.

Palavras-chave Má oclusão, Pré-escolar, Saúde bucal, Prevalência 


\section{Introdução}

As condições de saúde bucal dos pré-escolares brasileiros são preocupantes, pois embora tenham sido observadas discretas melhorias nos dois últimos levantamentos epidemiológicos realizados na população brasileira ${ }^{1,2}$, como o aumento em cerca de 6\% de crianças de 5 anos livres de cárie, tal contingente populacional ainda é acometido por história de doença não tratada, responsável por mais de $80 \%$ na composição do índice, apresenta diferentes necessidades de tratamento odontológico além da presença de problemas oclusais². A atenção odontológica nos primeiros anos de vida permite a identificação de fatores de risco para agravos e doenças bucais possibilitando o planejamento e a execução de procedimentos preventivos e curativos ${ }^{3}$, reduzindo assim o impacto desses problemas na vida diária desses indivíduos ${ }^{4}$.

Os problemas relacionados à oclusão são caracterizados por alterações no desenvolvimento dos maxilares podendo resultar em alterações funcionais, estéticas e psicossociais nos indivídu$\mathrm{os}^{5,6}$, sendo resultado de uma interação de fatores genéticos e ambientais ${ }^{7-9}$. Entre pré-escolares, os problemas relacionados à oclusão têm sido associados a diferentes fatores, tais como: déficit antropométrico ${ }^{10}$, hábitos deletérios, tais como o hábito de sucção de chupeta ou digital ${ }^{11}$, distúrbios da erupção dentária ${ }^{12}$ e a outros agravos bucais, como o traumatismo dentário ${ }^{13}$ e cárie dentária ${ }^{14}$. Além disso, a presença da má oclusão nessa faixa etária tem sido associada ao impacto negativo na qualidade de vida das crianças e de seus familiares $^{15}$. Estes achados têm sido oriundos de estudos de base populacional, contribuindo para o entendimento deste problema em pré-escolares.

Em geral, observa-se uma elevada prevalência de problemas oclusais entre pré-escolares, variando em diferentes países, com taxas próximas de 20\% na Espanha ${ }^{16}$ e de $70 \%$ na Lituânia ${ }^{17}$, como identificado em estudos populacionais prévios. No Brasil, a prevalência dos problemas oclusais em pré-escolares tem variado de $28 \%$ a $80 \%$, de acordo com a localidade da realização dos estudos ${ }^{11,13,15,18}$, o que ressalta a possibilidade da associação das características regionais na ocorrência deste agravo, atentando-se ao fato de que são diferentes os critérios de avaliação da má oclusão utilizados nestes estudos. Segundo dados dos dois últimos inquéritos nacionais de saúde bucal da população brasileira ${ }^{1,2}$, a presença de algum problema oclusal nas crianças de 5 anos aumentou em $28,2 \%$ entre os dois levantamentos ${ }^{12}$.
No entanto, não foram identificados na literatura estudos que tenham caracterizado o padrão epidemiológico de acometimento dos problemas oclusais entre pré-escolares, considerando uma amostra representativa desse contingente populacional no Brasil. Além disso, devido à variada prevalência desse agravo nos diferentes municípios brasileiros, apontadas por estudos prévios $^{11,13,15,18}$, a associação da má oclusão com a região geográfica de residência desses indivíduos necessita ser avaliada, assim como demais fatores associados. Nesta linha de raciocínio, diferenças regionais no padrão de acometimento podem nortear o planejamento de ações preventivas e corretivas direcionadas ao agravo. Portanto, o presente estudo, objetivou caracterizar o padrão de acometimento da má oclusão entre pré-escolares brasileiros, assim como identificar os fatores associados à sua presença, considerando como base os dados da Pesquisa Nacional de Saúde Bucal realizada em 2010 (SB Brasil).

\section{Métodos}

\section{Delineamento e amostragem}

Estudo transversal, analítico conduzido a partir da base de dados do levantamento epidemiológico das condições de Saúde Bucal da população brasileira realizado em $2010^{2}$.

O levantamento em questão foi conduzido seguindo os critérios propostos pela OMS, no qual uma amostra representativa da população brasileira nas faixas etárias índices foi entrevistada e examinada por meio de exames clínico-epidemiológicos realizados por cirurgiões-dentistas sob luz natural, em seus domicílios quanto às condições de saúde bucal, demográficas e socioeconômicas, uso de serviços odontológicos e questões subjetivas de saúde bucal.

A amostra foi composta a partir de residentes em 177 municípios brasileiros, das cinco macrorregiões brasileiras (Norte, Nordeste, Centro-Oeste, Sudeste e Sul), incluindo as 27 capitais, selecionados por amostragem probabilística por conglomerados, em múltiplos estágios, com probabilidade proporcional ao tamanho da população e considerando um efeito de desenho (deff) igual a 2,0. Os exames e entrevistas foram realizados por cirurgiões-dentistas previamente treinados e calibrados pela técnica do consenso, sendo o valor mínimo aceitável de Kappa para cada examinador, grupo etário e agravo estudado igual a $0,65^{19}$. 
No presente estudo considerou-se um recorte do banco de dados de crianças de 5 anos que foram examinadas quanto à maloclusão (7.045) excluindo-se aqueles de raça amarela (138) ou indígena (52), devido a baixa representatividade ${ }^{20}$, totalizando uma amostra de 6855 pré-escolares de 5 anos de idade.

\section{Avaliação da má oclusão}

A má oclusão foi diagnosticada segundo o Índice de Foster e Hamilton ${ }^{21}$ que é composto por quatro medidas: chave de caninos, sobressalência, sobremordida e mordida cruzada posterior. A medição (em milímetros) foi realizada com os dentes em oclusão e a sonda paralelamente ao plano oclusal. A criança que apresentou pelo menos uma dessas anormalidades foi caracterizada com "má oclusão", caso contrário, com "oclusão normal". No presente estudo adotou-se como variável dependente (desfecho) a presença de má oclusão.

\section{Variáveis independentes}

As variáveis independentes consideradas foram: macroregião (Norte, Centro-Oeste, Nordeste, Sudeste, Sul) localização geográfica (capital, interior), sexo (masculino, feminino) e raça autodeclarada (branca, negra/parda). Tais informações foram adquiridas por meio de questionários aplicados aos responsáveis pelos pré -escolares.

\section{Análise estatística e considerações éticas}

Inicialmente as variáveis foram descritas por meio de suas distribuições de frequências. Para as variáveis que compõem o Índice de Foster e Hamilton, bem como para o desfecho (má oclusão) foram estimados intervalo de $95 \%$ de confiança para suas respectivas prevalências. Na análise bivariada, utilizou-se teste do Qui-quadrado para avaliar a existência de associação entre o desfecho e as variáveis independentes. As variáveis que apresentaram nível descritivo (valor-p) menor ou igual a 0,20 foram selecionadas para $\mathrm{o}$ modelo múltiplo. Na análise múltipla adotou-se o modelo de regressão de Poisson, com variância robusta, utilizando a razão de prevalência (RP) como medida de associação. As análises estatísticas foram realizadas no programa SPSS. 18.0.

Olevantamento epidemiológico foi conduzindo respeitando os princípios éticos da Resolução do Conselho Nacional de Saúde nº196/96, sendo aprovado e registrado pela Comissão Nacional de Ética em Pesquisa (Conep).

\section{Resultados}

Foram incluídas no presente estudo 6855 pré-escolares de 5 anos de idade, sendo a maioria do sexo masculino, residentes nas regiões Nordeste e Norte do país e de cor de pele negra ou parda (Tabela 1).

Em relação a avaliação da má oclusão identificou-se que $4332(63,2 \%)$ dos pré-escolares possuíam pelo menos um dos problemas oclusais avaliados e, portanto, foram considerados com má oclusão. Os pré-escolares foram caracterizados quanto à presença de chave de caninos, sobressalência, sobremordida e mordida cruzada posterior (Tabela 2).

A análise bivariada demonstrou que apenas a macrorregião brasileira e o sexo se mantiveram associados à presença de má oclusão (Tabela 3).

$\mathrm{Na}$ análise múltipla de regressão de Poisson, identificou-se associações significativas da presença de má oclusão com a macrorregião brasileira $(\mathrm{p}=0,000$ a 0,026$)$ e com o sexo $(\mathrm{p}=0,003)$ (Tabela 4).

\section{Discussão}

O entendimento do padrão de acometimento da maloclusão nos pré-escolares brasileiros permite

Tabela 1. Distribuição das crianças de 5 anos segundo região, localização geográfica, sexo e cor de pele. Brasil, 2010.

\begin{tabular}{lrr}
\hline \multicolumn{1}{c}{ Variável } & n & $\%$ \\
\hline Região & & \\
Norte & 1687 & 24,6 \\
Centro-Oeste & 1091 & 15,9 \\
Nordeste & 1913 & 27,9 \\
Sudeste & 1248 & 18,2 \\
Sul & 916 & 13,4 \\
Localização & & \\
Capital & 5232 & 76,3 \\
$\quad$ Interior & 1623 & 23,7 \\
Sexo & & \\
$\quad$ Masculino & 3456 & 50,4 \\
$\quad$ Feminino & 3399 & 49,6 \\
Cor de pele & & \\
$\quad$ Branca & 3186 & 46,5 \\
$\quad$ Negra/Parda & 3669 & 53,5 \\
\hline
\end{tabular}


Tabela 2. Condição de oclusão dentária avaliada pelo índice de Foster e Hamilton na idade de 5 anos. Brasil, 2010.

\begin{tabular}{|c|c|c|c|}
\hline Condição de Oclusão & $\mathbf{n}^{*}$ & $\%$ & IC $_{95 \%}$ \\
\hline \multicolumn{4}{|l|}{ Chave de Caninos } \\
\hline Classe I & 5255 & 77,1 & $76,1-78,1$ \\
\hline Classe II & 1110 & 16,3 & $15,4-17,2$ \\
\hline Classe III & 451 & 6,6 & $6,0-7,2$ \\
\hline \multicolumn{4}{|l|}{ Sobressalência } \\
\hline Normal & 4214 & 67,1 & $65,9-68,3$ \\
\hline Aumentado & 1432 & 22,8 & $21,8-23,8$ \\
\hline Topo a topo & 438 & 7,0 & $6,3-7,6$ \\
\hline Cruzada Anterior & 196 & 3,1 & $2,7-3,6$ \\
\hline \multicolumn{4}{|l|}{ Sobremordida } \\
\hline Normal & 4085 & 65,4 & $64,2-66,6$ \\
\hline Reduzida & 795 & 12,7 & $11,9-13,6$ \\
\hline Aberta & 696 & 11,1 & $10,4-11,9$ \\
\hline Profunda & 673 & 10,8 & $10,0-11,5$ \\
\hline \multicolumn{4}{|l|}{ Mordida Cruzada Posterior } \\
\hline Ausência & 5532 & 81,3 & $80,3-82,2$ \\
\hline Presença & 1276 & 18,7 & $17,8-19,7$ \\
\hline \multicolumn{4}{|l|}{ Má Oclusão } \\
\hline Ausente & 2523 & 36,8 & $35,7-38,0$ \\
\hline Presente & 4332 & 63,2 & $62,1-64,3$ \\
\hline
\end{tabular}

Tabela 3. Padrão de oclusão dentária segundo região, localização, sexo e cor de pele em crianças de 5 anos de idade. Brasil, 2010.

\begin{tabular}{|c|c|c|c|c|c|}
\hline \multirow{2}{*}{ Variável } & \multicolumn{2}{|c|}{$\begin{array}{c}\text { Oclusão } \\
\text { normal }\end{array}$} & \multicolumn{2}{|c|}{ Má oclusão } & \multirow{2}{*}{ Valor- $\mathbf{p}^{*}$} \\
\hline & $\mathbf{n}$ & $\%$ & $\mathbf{n}$ & $\%$ & \\
\hline Região & & & & & $<0,001$ \\
\hline Norte & 772 & 45,8 & 915 & 54,2 & \\
\hline Centro-Oeste & 452 & 41,4 & 639 & 58,6 & \\
\hline Nordeste & 660 & 34,5 & 1253 & 65,5 & \\
\hline Sudeste & 391 & 31,3 & 857 & 68,7 & \\
\hline Sul & 248 & 27,1 & 668 & 72,9 & \\
\hline Localização & & & & & 0,247 \\
\hline Interior & 617 & 38,0 & 1006 & 62,0 & \\
\hline Capital & 1906 & 36,4 & 3326 & 63,6 & \\
\hline Sexo & & & & & 0,003 \\
\hline Masculino & 1332 & 38,5 & 2124 & 61,5 & \\
\hline Feminino & 1191 & 35,0 & 2208 & 65,0 & \\
\hline Cor de pele & & & & & 0,896 \\
\hline Preta/Parda & 1353 & 36,9 & 2316 & 63,1 & \\
\hline Branca & 1170 & 36,7 & 2016 & 63,3 & \\
\hline Total & 2523 & 36,8 & 4332 & 63,2 & \\
\hline
\end{tabular}

* teste do Qui-quadrado. estimar a característica da demanda regional por tratamento ortodôntico além do conhecimento do perfil destes indivíduos. Do ponto de vista da saúde pública, a identificação das diferenças sociodemográficas valoriza a necessidade de descentralização das políticas públicas de saúde bucal, o que possibilita a compreensão de que características regionais interferem na prevalência e severidade das doenças e agravos bucais. A prevalência da má oclusão entre os pré-escolares brasileiros, associada às macrorregiões brasileira e ao sexo dos pré-escolares, foi de $63,2 \%$, ou seja, esses indivíduos apresentaram pelos menos um dos problemas oclusais avaliados (chave de caninos, sobressaliência, sobremordida e/ou mordida cruzada anterior). Não tem sido bem esclarecido na literatura a caracterização do acometimento da má oclusão em crianças de 5 anos de idade considerando uma amostra representativa do Brasil, dificultando possíveis comparações. Resultados semelhantes de prevalência de má oclusão foi identificada entre crianças da Alemanha $(72 \%)^{12}$ e Lituânia $(71 \%)^{17}$.

Ao considerar estudos realizados em diferentes regiões do Brasil apontando características locais de acometimento, observa-se uma prevalência bastante variável. Prevalência semelhante a registrada no presente estudo foi identificada em estudo prévio realizado em Bauru (SP) ${ }^{11}$, com acometimento em 50 e $60 \%$ entre crianças de 3 a 5 anos, utilizando critério diagnóstico diferente. Em contrapartida, estudo prévio, realizado em outro estado (MG), identificou uma prevalência inferior, com taxa de $28,4 \%$, utilizando o critério de Foster e Hamilton em crianças de 3 a 5 anos de idade ${ }^{15}$. Já prevalência superior foi identificada em município da região Sul (Canoas- RS), com taxa de $69,9 \% \%^{4}$, considerando diferente critério de diagnóstico. As diferenças regionais, assim como os diferentes critérios de diagnostico utilizados nos estudos podem explicar a alta variabilidade na prevalência entre os diferentes locais no Brasil. Apesar disto, considerando o impacto negativo desse agravo na qualidade de vida de pré-escolares e de seus familiares ${ }^{15}$, assim como a possibilidade da presença desses problemas na dentição decídua acarretar em uma maior necessidade de tratamento ortodôntico na dentição permanente ${ }^{22}$, a taxa de prevalência encontrada é preocupante.

Em adição, identificou-se a associação entre a presença da má oclusão com a região geográfica e o sexo dos pré-escolares, partir da análise ajustada. Maior prevalência foi identificada entre os pré -escolares residentes nas regiões Centro-Oeste, Nordeste, Sudeste e Sul, em ordem crescente da 
Tabela 4. Análise da associação entre prevalência de má oclusão dentária segundo região, localização, sexo e cor de pele em crianças de 5 anos. Brasil, 2010.

\begin{tabular}{|c|c|c|c|c|}
\hline \multirow{2}{*}{ Variável } & \multicolumn{2}{|c|}{ Análise bruta } & \multicolumn{2}{|c|}{ Análise ajustada } \\
\hline & RP $\left(\right.$ IC $\left._{95 \%}\right)$ & Valor-p & $\mathbf{R P}\left(\mathbf{I C}_{95 \%}\right)$ & Valor-p \\
\hline \multicolumn{5}{|l|}{ Região } \\
\hline Norte & 1,00 & & 1,00 & \\
\hline Centro-Oeste & $1,08(1,01-1,20)$ & 0,023 & $1,08(1,01-1,15)$ & 0,026 \\
\hline Nordeste & $1,21(1,14-1,28)$ & $<0,001$ & $1,21(1,14-1,28)$ & $<0,001$ \\
\hline Sudeste & $1,27(1,20-1,34)$ & $<0,001$ & $1,27(1,20-1,34)$ & $<0,001$ \\
\hline Sul & $1,35(1,27-1,43)$ & $<0,001$ & $1,34(1,26-1,42)$ & $<0,001$ \\
\hline \multicolumn{5}{|l|}{ Localização } \\
\hline Interior & 1,00 & & & \\
\hline Capital & $1,03(0,98-1,07)$ & 0,247 & n.s & n.s \\
\hline \multicolumn{5}{|l|}{ Sexo } \\
\hline Masculino & 1,00 & & 1,00 & \\
\hline Feminino & $1,06(1,02-1,10)$ & 0,003 & $1,06(1,02-1,09)$ & 0,003 \\
\hline \multicolumn{5}{|l|}{ Cor de pele } \\
\hline Preta/Parda & 1,00 & & & \\
\hline Branca & $1,00(0,97-1,04)$ & 0,896 & n.s & n.s \\
\hline
\end{tabular}

chance de acometimento, quando comparados à região Norte do país. Diferenças semelhantes entre as regiões brasileiras no acometimento da má oclusão foram também identificadas em escolares $(12 \text { anos })^{23}$ e adolescentes brasileiros (15 a 19 anos $)^{24}$. Outras doenças bucais em pré-escolares, como a cárie dentária, também têm demonstrado diferenças de acometimento de acordo com a região geográfica do $\mathrm{Brasil}^{25}$. Considerando a dimensão do Brasil em termos territoriais e a existência de diferenças regionais e culturais, é possível que comportamentos em saúde sejam influenciados por estas diferenças e, consequentemente, impactem nos desfechos em saúde, tal como a má oclusão. Entre estas diferenças regionais e culturais existentes, destacam-se: o nível socioeconômico das famílias e dos municípios, que pode influenciar no acesso a serviços odontológicos; o nível educacional dos residentes das regiões, podendo influenciar nos comportamentos; diferenças comportamentais devido à questões culturais que podem levar à ocorrência de problemas oclusais.

Diferenças comportamentais entre residentes das diferentes regiões do Brasil podem ser exemplificadas pelo padrão de uso de serviços de saúde pelo país ${ }^{2,26}$. Além disso, alguns comportamentos têm sido indicados como fatores de risco para a ocorrência de problemas oclusais ${ }^{11}$ e estes podem variar de acordo com diferenças culturais, ou seja, diferentes regiões podem levar a diferentes comportamentos que podem impactar na ocorrência de má oclusão. Em adição, o perfil de acometimento dos problemas bucais ${ }^{2}$, assim como o acesso e o processo de atenção à saúde podem apresentar diferenças entre as regiões brasileiras ${ }^{27}$, o que pode impactar em uma maior ocorrência de agravos bucais. Dessa forma, as ações de saúde devem considerar tais diferenças, com intuito de atender apropriadamente a população.

Identificou-se ainda que a presença da má oclusão foi maior entre pré-escolares do sexo feminino, o que também foi identificado em estudo prévio conduzido em município da região Sudeste (Bauru - SP) ${ }^{11}$. Associação entre má oclusão e sexo, com maior chance de ocorrência entre meninas, foi identificada também entre adolescentes brasileiros ${ }^{24}$ e indianos ${ }^{28}$. Resultado contrário foi identificado para crianças brasileiras aos 12 anos de idade, com uma maior prevalência da má oclusão no sexo masculino ${ }^{29}$. Assim como para as diferenças entre as regiões brasileiras, sabe-se que questões sociodemográficas podem influenciar na ocorrência dos desfechos em saúde bucal, tal como a má oclusão. Ressalta-se que, apesar da associação identificada, no campo da saúde o sexo feminino tem sido considerado um fator protetor, com menor ocorrência de agravos ${ }^{23}$. Uma possível explicação para a di- 
ferença na associação com sexo entre as idades de 5 e 12 anos é o período da vida em que estas crianças se encontram. Aos 5 anos as condições de saúde, assim como comportamentos, são, em sua maioria, moduladas pelas ações dos responsáveis, ou seja, é um reflexo do cuidado ou da importância que o responsável tem sobre a criança, já que é uma idade bastante precoce, não tendo independência em relação a saúde. Portanto, essa maior ocorrência no sexo feminino não necessariamente representa um perfil de risco. Já aos 12 anos a condição de saúde se torna mais reflexo dos comportamentos e personalidade da própria criança. Outro fator a ser considerado é o valor da razão de prevalência identificada para o sexo feminino no presente estudo $(1,06)$, sendo próximo a 1, demonstrando que apesar da diferença entre os sexos existir, a chance de ocorrência da má oclusão é bastante próxima entre meninos e meninas, ou sejam, as meninas têm somente $6 \%$ maior probabilidade de ter o desfecho em comparação aos meninos.

A caracterização do padrão epidemiológico de acometimento e possíveis fatores associados a má oclusão entre pré-escolares brasileiros pode subsidiar ações de saúde e melhoria nas políticas públicas de saúde bucal que visem a redução desse agravo. Além disso, considerando que as crianças em fase pré-escolar se configuram como um contingente populacional mais propenso a aceitar novos hábitos, a educação em saúde nessa fase, assim como de seus familiares, pode ser uma alternativa eficaz para impactar positivamente nos desfechos em saúde buca ${ }^{30}$. Portanto, salienta-se a necessidade de criação e ou otimização das atividades educativas de caráter permanen$\mathrm{te}^{30}$, a fim de reduzir fatores de risco a má oclusão.

Os problemas oclusais podem se desenvolver cedo, em idades precoces, e dificilmente podem ser autocorrigidos, o que torna a fase da dentição decídua um período adequado para a inserção de medidas preventivas ou de tratamento. Além disso, considerando o impacto negativo da má oclusão na qualidade de vida da criança, o que pode impactar no contexto familiar, torna-se necessário a resolução deste problema o mais precoce possível. Dentre as condições oclusais avaliadas, observou-se um relevante acometimento de classe II de caninos, sobressaliência aumenta- da e mordida cruzada posterior. Estudos clínicos têm abordado diferentes modalidades terapêuticas para o tratamento de problemas oclusais em crianças na idade pré-escolar, assim como medidas intervencionais para controle de possíveis fatores de risco ${ }^{31,32}$. Tem-se como exemplo estudo de Lippold et al..$^{32}$ que indicou expansão maxilar inicial seguida de terapia ativadora com arco de U, para tratamento de mordida cruzada posterior em crianças com dentição decídua ou mista, a partir de estudo clínico ${ }^{32}$ Em adição, o uso de intervenções psicológicas e uso de aparelhos ortodônticos, como o arco palatino, têm demonstrado serem efetivos na cessação de hábitos de sucção não-nutritivos que poderiam acarretar em má oclusão entre crianças, como demonstrado por revisão sistemática da literatura ${ }^{31}$.

Nesta linha, no Brasil, os Centros de Especialidades Odontológicas (CEO) podem incluir no rol de especialidades, desde 2011, o tratamento ortodôntico corretivo, no entanto, os aparelhos ortopédicos e ortodônticos removíveis podem ser realizados tanto na atenção básica quanto nos $\mathrm{CEOs}^{33}$, procedimentos estes que seriam de grande valia para a correção precoce das maloclusões contribuindo assim para melhoria da saúde bucal de parcela significante dos pré-escolares brasileiros.

$\mathrm{O}$ presente estudo não permite estabelecer relações de causa e efeito entre os fatores estudados. Portanto, estudos futuros, principalmente de caráter longitudinal, podem esclarecer a possível influência dos fatores associados na ocorrência dos problemas oclusais em pré-escolares brasileiros. Em adição, considerando as relevantes desigualdades sociais presentes no Brasil, torna-se necessário a avaliação da possível influência de aspectos socioeconômicos na ocorrência deste agravo. Apesar disso, os resultados apresentados são válidos e representativos da população de uma amostra representativa de pré-escolares de 5 anos de idade do Brasil, permitindo mais esclarecimentos sobre a prevalência e fatores sociodemográficos associados a má oclusão. A partir dos dados, identificou-se uma alta prevalência da má oclusão entre os pré-escolares brasileiros investigados, sendo esta prevalência maior entre aqueles do sexo feminino e diferenciando de acordo com as macrorregiões brasileiras. 


\section{Colaboradores}

JM Bauman e FM Flório participaram da organização e análise dos dados, redação do artigo, revisão crítica e aprovação da versão submetida. JGS Souza e CD Bauman participaram da organização dos dados e redação do artigo.

\section{Referências}

1. Brasil. Ministério da Saúde (MS). Projeto SB Brasil 2003: condições de saúde bucal da população brasileira 2002-2003: resultados principais. Brasília: MS; 2004.

2. Brasil. Ministério da Saúde (MS). Projeto SB Brasil 2010: condições de saúde bucal da população brasileira, resultados principais. Brasília: MS; 2011.

3. Rodrigues LAM, Martins AMEBL, Silveira MF, Ferreira RC, Souza JGS, Silva JM, Caldeira AP. Uso de serviços odontológicos entre pré-escolares: estudo de base populacional. Cien Saude Colet 2014; 19(10):4247-4256.

4. Kramer PF, Feldens CA, Ferreira SH, Bervian J, Rodrigues PH, Peres MA. Exploring the impact of oral diseases and disorders on quality of life of preschool children. Community Dent Oral Epidemiol 2013; 41(4):327-335.

5. Peres KG, Tomita NE. Oclusopatias. In: Antunes JLF, Peres MA. In: Epidemiologia da saúde bucal. Rio de Janeiro: Guanabara Koogan; 2008. p. 83-101.

6. Peres KG, Barros AJD, Anselmi L, Peres MA, Barros FC. Does malocclusion influence the adolescent's satisfaction with appearance? A cross-sectional study nested in a Brazilian birth cohort. Community Dent Oral Epidemiol. 2008; 36(2):137-143.

7. Shaw WC, Addy M, Ray C. Dental and social effects of malocclusion and effectiveness of orthodontic treatment: a review. Community Dent Oral Epidemiol. 1980; 8(1):36-45.

8. Perillo L, Monsurro A, Bonci E, Torella A, Mutarelli M, Nigro V. Genetic Association of ARHGAP21 Gene Variant with Mandibular Prognathism. J Dent Res. 2015; 94(4):569-576.

9. Petersen PE. The World Oral Health Report 2003: continuous improvement of oral health in the 21st century--the approach of the WHO Global Oral Health Programme. Community Dent Oral Epidemiol 2003; 31(Supl. 1):3-23.

10. Thomaz EBAF, Valença AMG. Associação entre déficit de peso e apinhamento na dentição decídua. J Pediatr 2009; 85(2):110-116.

11. Tomita NE, Bijella VT, Franco LJ. Relação entre hábitos bucais e má oclusão em pré-escolares. Rev Saude Publica 2000; 34(3):299-303.

12. Salbach A, Schremmer B, Grabowski R, Stahl de Castrillon F. Correlation between the frequency of eruption disorders for first permanent molars and the occurrence of malocclusions in early mixed dentition. $J$ Orofac Orthop 2012; 73(4):298-306.

13. Goettems ML, Azevedo MS, Correa MB, Costa CT, Wendt FP, Schuch HS, Bonow ML, Romano AR, Torriani DD. Dental trauma occurrence and occlusal characteristics in Brazilian preschool children. Pediatr Dent 2012; 34(2):104-107.

14. Marquezan M, Faraco-Junior IM, Feldens CA, Kramer PF, Ferreira SH. Association between occlusal anomalies and dental caries in 3- to 5 year-old Brazilian children. J Orthod 2011; 38(1):8-14.

15. Ramos-Jorge J, Motta T, Marques LS, Paiva SM, Ramos-Jorge ML. Association between anterior open bite and impact on quality of life of preschool children. Braz Oral Res 2015; 29(1):1-7. 
16. Cardoso Silva C, Maroto Edo M, Soledad Alvaro Llorente M, Barbería Leache E. Primary molar infraocclusion: frequency, magnitude, root resorption and premolar agenesis in a Spanish sample. Eur J Paediatr Dent 2014; 15(3):258-264.

17. Kasparaviciene K, Sidlauskas A, Zasciurinskiene E, Vasiliauskas A, Juodzbalys G, Sidlauskas M, Marmaite $\mathrm{U}$. The prevalence of malocclusion and oral habits among 5-7-year-old children. Med Sci Monit 2014; 20:2036-2042.

18. Hebling SRF, Cortellazzi KL, Tagliaferro EPS, Hebling E, Ambrosano GMB, Meneghim MC, Pereira AC. Relationship between malocclusion and behavioral, demographic and socioeconomic variables: a cross-sectional study of 5-year-olds. J Clin Pediatr Dent 2008; 33(1):75-80.

19. Roncalli AG, Silva NN, Nascimento AC, Freitas CHSM, Casotti E, Peres KG, Moura L, Peres MA, Freire MCM, Cortes MIS, Vettore MV, Junior MP, Figueiredo N, Goes PSA, Pinto RS, Marques RAA, Moyses SJ, Reis SCGB, Narvai PC. Aspectos metodológicos do Projeto SBBrasil 2010 de interesse para inquéritos nacionais de saúde. Cad Saude Publica 2012; 28(Supl.):s40-s57.

20. Peres KG, Frazão P, Roncalli AG. Padrão epidemiológico das oclusopatias muito graves em adolescentes brasileiros. Rev Saude Publica 2013; 47(Supl. 3):109-117.

21. Foster TD, Hamilton MC. Occlusion in the primary dentition: study of children at 2 and one-half to 3 years of age. Br Dent J 1969; 126(2):76-79.

22. Peres KG, Peres MA, Thomsom WM, Broadbent J, Hallal PC, Menezes AB. Deciduous-dentition malocclusion predicts orthodontic treatment needs later: Findings from a population-based birth cohort study. Am J Orthod Dentofacial Orthop 2015; 147(4):492-498.

23. Bauman JM, Souza JGS, Bauman CD, Flório FM. Aspectos sociodemográficos relacionados à gravidade da maloclusão em crianças brasileiras de 12 anos. Cien Saude Colet 2018; 23(3):723-732.

24. Freitas CV, Souza JGS, Mendes DC, Pordeus IA, Jones KM, Martins AMEBL. Necessidade de tratamento ortodôntico em adolescentes brasileiros: avaliação com base na saúde pública. Rev Paul Pediatr 2015; 33(2):204-210.

25. Ardenghi TM, Piovesan C, Antunes JLF. Desigualdades na prevalência de cárie dentária não tratada em crianças pré-escolares no Brasil. Rev Saude Publica 2013; 47(Supl. 3):129-137.

26. Pinheiro RS, Torres TZG. Uso de serviços odontológi- cos entre os estados do Brasil. Cien Saude Colet 2006; 11(4):999-1010.

27. Roncalli AG, Cortes MIS, Peres KG. Perfis epidemiológicos de saúde bucal no Brasil e os modelos de vigilância. Cad Saude Publica 2012; 28(Supl.):S58-S66.

28. Sanadhya S, Chadha M, Chaturvedi MK, Chaudhary M, Lerra S, Meena MK, Bakutra G, Acharya S, Pandey A, Tak M, Asawa K, Kamate S. Prevalence of malocclusion and orthodontic treatment needs among 12-15-year-old schoolchildren of fishermen of Kutch coast, Gujarat, India. Int Marit Health 2014; 65(3):106113.

29. Brizon VSC, Cortellazzi KL, Vazquez FL, Ambrosano GMB, Pereira AC, Gomes VE, Oliveira AC. Fatores individuais e contextuais associados à má oclusão em crianças brasileiras. Rev Saude Publica 2013; 47(Supl. 3):118-128.

30. Oliveira RCN, Souza JGS, Oliveira CC, Oliveira LFB, Pelino JEP, Martins AMEBL, Almeida ER. Acesso a informações sobre como evitar problemas bucais entre escolares da Rede Pública de Ensino. Cien Saude Colet 2015; 20(1):85-94.

31. Borrie FR, Bearn DR, Innes NP, Iheozor-Ejiofor Z. Interventions for the cessation of non-nutritive sucking habits in children. Cochrane Database Syst Rev 2015; 3:CD008694.

32. Lippold C, Stamm T, Meyer U, Vegh A, Moiseenko T, Danesh G. Early treatment of posterior crossbite-a randomised clinical trial. Trials 2013; 14:20.

33. Brasil. Ministério da Saúde (MS). Saúde de Família: passo a passo do departamento de atenção básica. Brasilia: MS; 2011.

Artigo apresentado em 28/06/2016

Aprovado em 12/11/2016

Versão final apresentada em 14/11/2016 